\title{
ON LEVI-CIVITA'S THEORY OF PARALLELISM
}

\author{
BY C. E. WEATHERBURN
}

1. Parallel Displacements. The use of two-parametric differential invariants* (grad, div etc.) for a surface not only leads very neatly and quickly to the condition of parallelism and the known theorems, but also puts in evidence other results hitherto unnoticed. In particular, some interesting properties of Tchebychef nets are discovered.

Consider a curve $C$ drawn on a surface $S$ in ordinary space. Let $R$ be a vector of constant length (which may be taken as unity), and a point-function along $C$, being everywhere tangential to the surface. Then $R$ is said to undergo a parallel displacement along $C$, or to remain parallel to itself for displacement along this curve, provided the derivative of $\boldsymbol{R}$ along $C$ is everywhere normal to the surface. $\dagger$ We lose no generality by regarding $C$ as a member of a family $T$ of curves, with unit tangent $t$ and arc-length $s$, and $R$ as the unit tangent to a second family cutting the former at a variable angle $\theta$, so that $\theta$ is the angle of rotation from $t$ to $\boldsymbol{R}$ in the positive sense about the unit normal $\boldsymbol{n}$. If then $m$ is the unit surface vector $n \times t$, perpendicular to $t$, we may write

$$
R=t \cos \theta+m \sin \theta .
$$

Let a prime denote differentiation with respect to $s$, that is to say, in the direction of $t$. Then in order that $R$ may be normal to $S$ we must have

and

$$
0=t \cdot R^{\prime}=\sin \theta\left[t^{\prime} m \cdot-\theta^{\prime}\right]
$$

$$
0=m \cdot R^{\prime}=\cos \theta\left[m \cdot t^{\prime}+\theta^{\prime}\right],
$$

* All the differential invariants of this article are two-parametric invariants. See the writer's Differential Geometry, Chap. 12, Cambridge University Press, or Quarterly Journal of Mathematics, vol. 50, pp. 230269.

† See Levi-Civita, The Absolute Differential Calculus, pp. 101-119. 
from either of which it follows that

$$
\frac{d \theta}{d s}=\operatorname{div} \boldsymbol{m}=-\gamma
$$

where $\gamma$ denotes* the geodesic curvature of $C$. This is Levi-Civita's formula $\dagger$ for the arc-rate of increase of $\theta$ along $C$. From it follow the theorems of the constancy of the angle between two vectors undergoing parallel displacements along $C$, and of the constancy of $\theta$ for parallel displacement along a geodesic.

Since the derivative of $R$ along $C$ is normal to $S$, its magnitude is given by

$$
n \cdot R^{\prime}=n \cdot\left(t^{\prime} \cos \theta+m^{\prime} \sin \theta\right)=\kappa_{n} \cos \theta+\tau \sin \theta,
$$

where $\kappa_{n}$ is the normal curvature of the surface in the direction of $t$, and $\tau$ is the torsion of the geodesic tangent to $C$, or the moment $\ddagger$ of the family of curves $T$. This magnitude is clearly the resolved part of the vector $k_{n} t+\tau m$ in the direction of $R$.

Again, consider a family of curves $A$ with unit tangent a, and their orthogonal trajectories $B$ with unit tangent $\boldsymbol{b}$, so that $n=a \times b$. Let a third family $T$ cut the first at a variable angle $-\theta$, so that the unit tangent $t$ to this family is given by $t=a \cos \theta-b \sin \theta$. Then the geodesic curvature $\gamma$ of the last family is found from $\S$

$$
\begin{aligned}
-\gamma & =\operatorname{div}(a \sin \theta+b \cos \theta) \\
& =\sin \theta \operatorname{div} a+\cos \theta \operatorname{div} b+t \cdot \nabla \theta,
\end{aligned}
$$

where $\nabla \theta$ denotes the gradient of $\theta$. If now the vector $a$ is such that it suffers a parallel displacement along a curve of the family $T$, we have $t \cdot \nabla \theta=-\gamma$, and (3) becomes

$$
\sin \theta \operatorname{div} a+\cos \theta \operatorname{div} b=0,
$$

\footnotetext{
* See the writer's Differential Geometry, p. 234.

$\dagger$ Levi-Civita, Palermo Rendiconti, vol. 42 (1917), p. 185.

$\$$ Differential Geometry, \$130.

§ Ibid., \$121.
} 
from which two theorems may be deduced. First suppose that the curves of the family $A$ constitute a family of parallels. Then $\operatorname{div}$ a vanishes* identically; and the last equation shows that either $\cos \theta=0$ or $\operatorname{div} b=0$. The second of these alternatives can be satisfied only where the geodesic curvature of the parallel curves is zero, that is to say, on their line of normal curvature. Hence we have the following theorem.

THEOREM 1. If the tangent to a family of parallel curves undergoes a parallel displacement along a transversal, either this transversal is an orthogonal trajectory (and therefore a geodesic), or else it is a line of normal curvature of the family of parallels.

Secondly, let the family $A$ be one of geodesics. Then $\operatorname{div} b$ vanishes identically, and the previous equation $\left(3^{\prime}\right)$ takes the form $\sin \theta \operatorname{div} a=0$. Since then $\sin \theta$ is not zero, this requires $\operatorname{div} a=0$, which holds only at the line of striction $\dagger$ of the family of geodesics. Hence we have Bianchi's theorem $\ddagger$ that if the tangent to a family of geodesics on a surface undergoes a parallel displacement along a transversal this transversal is a line of striction of the family of geodesics.

From (3) follows also another result which, though not connected with the parallelism of Levi-Civita, may be mentioned here and will be used in $\$ 2$. Suppose that the two families $T$ and $A$ are geodesics, and that the angle $\theta$ is constant along each member of the former. Then $\gamma$, $\operatorname{div} \boldsymbol{b}$ and $t \cdot \nabla \theta$ all vanish identically, and therefore by (3), so also does div a. The family $A$ is thus also a family of parallels; and the surface is developable. For the second (or Gaussian) curvature $K$ is given by $\S$

$$
K=-(\operatorname{div} \boldsymbol{a} \operatorname{div} \boldsymbol{a}+\boldsymbol{b} \operatorname{div} \boldsymbol{b})=0 .
$$

* Differential Geometry, p. 258.

$\dagger$ Ibid., \$126.

$\ddagger$ Geometria Differenziale, vol. 2, p. 802.

$\S$ Differential Geometry, \$125. 
Hence we have the following theorem:

THEOREM 2. If a family of geodesics cut a family of geodesics (or parallels) at an oblique angle which is constant along each member of the former, the latter are both geodesics and parallels, and the surface is developable.

2. Tchebychef Nets. A Tchebychef system (or net) on a given surface consists of two families of curves, $U, V$, such that the tangent to each family undergoes a parallel displacement along each curve of the other family.* If $u, v$, are the unit tangents to the two families $U, V$, respectively, the derivative of $v$ in the direction of $u$ is $u \cdot \nabla v$; and since this is normal to the surface we must have $u \cdot \nabla v \cdot u=0$. Similarly it follows that $v \cdot \Delta u \cdot v=0$. Conversely, if these relations hold, both derivatives are normal to the surface, and we $h$ ave the following theorem.

TheOREM 3. Necessary and sufficient conditions that the two families of curves with unit tangents $u, v$ constitute a Tchebychef system are expressed by the equations

$$
u \cdot \nabla v \cdot u=0, \quad v \cdot \nabla u \cdot v=0 .
$$

Now the tendency of a family of curves in any direction is the resolved part in that direction of the derivative of the unit tangent in that direction. $\dagger$ The equations (5) therefore express that, at any point of the surface, the tendency of each family in the direction of the other is zero. If then we introduce the conics

$$
\boldsymbol{r} \cdot \nabla \boldsymbol{u} \cdot \boldsymbol{r}=1
$$

and

$$
\boldsymbol{r} \cdot \nabla \boldsymbol{v} \cdot \boldsymbol{r}=1
$$

* Bianchi, loc. cit., p. 809.

† See $\$ 1$ of a paper by the author, On some properties of a family of curves on a surface, recently communicated to the Edinburgh Mathematical Society.

$\ddagger$ For this form of the equation of a quadric see the author's Advanced Vector Analysis, G. Bell and Sons, $\$ 66$. 
whose centers are at $P$, the origin of the vector $\boldsymbol{r}$, and whose plane is the tangent plane at $P$, it follows from (5) that $v$ has the direction of one of the asymptotes of (6). But the other asymptote has the direction of $u$; so that the tangents to the two families of curves are the asymptotes of (6). The same is true of the conic (7), and the two conics are therefore similar. The tendency of the family $U$ in any direction varies inversely as the square of the radius of the conic (6) in that direction.* Thus the ratio of the tendencies of the two families in any direction at $P$ is invariant. But the sum of the tendencies of a family in two directions at right angles is equal to the divergence of the family. Hence we may state the following theorem.

THEOREM 4. The ratio of the tendencies of the two families of a Tchebychef system in any direction at a given point is invariant, and equal to the ratio of their divergences at that point.

Also, at points where the two families $U$ and $V$ cut orthogonally the asymptotes of the conics (6) and (7) are at right angles. The sum of the tendencies in two perpendicular directions is zero for each family, so that the divergence of each is zero. Hence we have the following theorem.

THEOREM 5. The locus of the points at which the two families of a Tchebychef system cut orthogonally is a common line of striction for the two families.

Let $p, q$ denote the unit tangential vectors $n \times u, n \times v$ perpendicular to $u, v$ respectively. Then the geodesic curvatures $\gamma_{1}, \gamma_{2}$ of the two curves $U, V$ are given by the equations

$$
-\gamma_{1}=\operatorname{div} \boldsymbol{p}, \quad-\gamma_{2}=\operatorname{div} \boldsymbol{q} \text {. }
$$

Let $\theta$ be the angle of rotation from $u$ to $v$ in the positive sense. Then, since the vector $u$ undergoes a parallel displacement along the curve $V$, it follows from (3) and $\left(3^{\prime}\right)$ that

$$
\operatorname{div} u \tan \theta=\operatorname{div} \boldsymbol{p}=-\gamma_{1} .
$$

\footnotetext{
* Edinburgh Mathematical Society, loc. cit., \$1.
} 
From equation (8), $\gamma_{1}=0$ implies div $u=0$ since $\tan \theta \neq 0$. We may therefore state the following theorem.

THEOREM 6. A line of normal curvature of either family of a Tchebychef system is also a line of striction of that family.

The gradient of $\theta$ may be expressed in terms of $u$ and $v$ as follows. Taking the rot of both members of the identity

$$
u \times v=n \sin \theta,
$$

we have*

(10) $\boldsymbol{v} \cdot \nabla \boldsymbol{u}-\boldsymbol{u} \cdot \nabla \boldsymbol{v}+\boldsymbol{u} \operatorname{div} \boldsymbol{v}-\boldsymbol{v} \operatorname{div} \boldsymbol{u}=\cos \theta \nabla \theta \times \boldsymbol{n}$.

The derivatives represented by the first two terms are parallel to $n$. Therefore, on forming the vector product of both sides with $n$, we have*

$$
\cos \theta \nabla \theta=n \times(u \operatorname{div} v-v \operatorname{div} u) .
$$

Since $\cos \theta=u \cdot v$, this formula expresses $\nabla \theta$ in terms of $u$ and $v$. Where $\cos \theta$ is zero, both $\operatorname{div} u$ and $\operatorname{div} v$ must vanish, and we see again that this is a common line of striction of the two families $U$ and $V$.

If the two families of a Tchebychef net cut at a constant angle, $\nabla \theta$ is everywhere zero, so that $\operatorname{div} u$ and $\operatorname{div} v$ must vanish identically. Thus both families are families of parallel curves; and, as they cut at a constant angle, it follows from the last theorem of $\$ 1$ that they are also geodesics, and the surface is developable. Hence we have the following theorem.

THEOREM 7. If the two families of a Tchebychef net cut at a constant angle, each is a family of parallel geodesics, and the surface is developable.

Canterbury College,

Christchurch, New Zealand

*Differential Geometry, p. 237, Ex. 10. 